\title{
Geometric Curve Modeling with Sobolev Gradients
}

\author{
Robert J. Renka \\ Dept. of Computer Science \& Engineering \\ University of North Texas \\ Denton, Texas
}

July 20, 2006

\begin{abstract}
The Sobolev gradient method is a powerful tool for geometric modeling. We treat the problem of constructing fair curves by minimizing a fairness measure subject to geometric constraints. The measure might include curve length, curvature, torsion, and/or variation of curvature. The constraints may include specified values, tangent vectors, and/or curvature vectors. We may also require periodicity in the case of closed curves, or nonlinear inequalities representing shape-preservation criteria. The curve is represented by discrete vertices and divided difference approximations to derivatives with respect to arc length. A Sobolev gradient method is then particularly effective for minimizing the functional.
\end{abstract}

\section{Introduction}

Our first application of the Sobolev gradient method to a geometric modeling problem involved the construction of a surface with minimal surface area and constrained to pass through a space curve ([2]). The analogous curve-fitting problem is the construction of the minimum-length curve that passes through a pair of points. The problem is trivial in that the solution is just the line segment defined by the endpoints, but as a numerical optimization problem, it is not uninteresting. We will show that standard methods are doomed to failure, while a generalization of the Sobolev gradient method produces the solution in a single iteration. The latter observation was the starting point for our work in [2].

We restrict attention here to the simple minimum-length curve problem. While this problem is sufficient to demonstrate the key ideas, more complex applications involve a number of algorithmic details that can strongly affect the efficiency of the method, and we refer the reader to the literature for more extensive discussion. In [3] we treated the problem of interpolation with nonlinear splines, and in [4] we discussed the more general problem of construct- 
ing parametric space curves that minimize variation of curvature and take on pre-specified values, tangent vectors, and curvature vectors. The problem of constructing elastic curves constrained to lie in a regular surface is treated in [5], and [1] is addressed to the construction of periodic closed geodesics in a regular surface. Finally, in [6] we extended the work of [4] to handle inequalities representing shape-preservation criteria.

In the following section we discuss the minimum curve-length problem in the function space setting. Section 3 is addressed to the discretized problem, and test results are presented in Section 4.

\section{Minimum Curve-length}

For the problem treated here the distinction between planar curves and space curves is not important. For $n=2$ or $n=3$ the set of $C^{2}$ regular parametric curves in $\mathbf{R}^{n}$ is

$$
\mathcal{C}=\left\{f \in\left(C^{2}[0,1]\right)^{n}: f^{\prime}(t) \neq 0 \quad \forall t \in[0,1]\right\}
$$

We also need a space of perturbations which preserve the endpoint values of elements of $\mathcal{C}$ :

$$
\mathcal{C}_{o}=\left\{h \in\left(C^{2}[0,1]\right)^{n}: h(0)=h(1)=0\right\} .
$$

The curve length functional is $\phi: \mathcal{C} \rightarrow \mathbf{R}$ defined by

$$
\phi(f)=\int_{0}^{1}\left\|f^{\prime}(t)\right\| d t=\int_{0}^{1} s^{\prime}(t) d t
$$

where $s$ is arc length associated with $f$. We may think of the curve as a trajectory of a moving particle, in which case $t$ represents time, $f^{\prime}(t)$ is a velocity vector tangent to the curve at $f(t), s(t)$ is the arc length traversed between times 0 and $t$, and $s^{\prime}(t)$ is speed. The curvature vector is denoted $\kappa N$, where $N$ is a unit vector orthogonal to $f^{\prime}$ and in the plane defined by the velocity $f^{\prime}$ and acceleration $f^{\prime \prime}$ :

$$
\kappa N=\frac{f^{\prime} \times f^{\prime \prime} \times f^{\prime}}{\left\|f^{\prime}\right\|^{4}} .
$$

The above expression is easily derived from the alternative definition of curvature as the second derivative of $f$ with respect to arc length $s$. With the standard abuse of notation:

$$
\kappa N=\frac{d^{2} f}{d s^{2}}=\frac{d}{d s}\left(\frac{f^{\prime}}{s^{\prime}}\right)=\frac{1}{s^{\prime}}\left(\frac{f^{\prime}}{s^{\prime}}\right)^{\prime}=\frac{1}{\left\|f^{\prime}\right\|}\left(\frac{f^{\prime}}{\left\|f^{\prime}\right\|}\right)^{\prime} .
$$

The set of regular curves $\mathcal{C}$ has no zero element, and is therefore not a linear space. Rather it is an infinite-dimensional manifold. In order to define a metric on $\mathcal{C}$, we must define an inner product on the tangent space at each point $f \in \mathcal{C}$. Using the $L_{2}$ inner product, the $L_{2}$ gradient $\nabla \phi(f)$ is defined by 
$\phi^{\prime}(f) h=\langle\nabla \phi(f), h\rangle_{\left(L_{2}[0,1]\right)^{n}} \quad \forall h \in \mathcal{C}_{o}$, where the Fréchet derivative of $\phi$ at $f$ in the direction $h$ is

$$
\begin{aligned}
\phi^{\prime}(f) h & =\lim _{\alpha \rightarrow 0} \frac{\phi(f+\alpha h)-\phi(f)}{\alpha}=\lim _{\alpha \rightarrow 0} \frac{1}{\alpha} \int_{0}^{1}\left(\left\|f^{\prime}+\alpha h^{\prime}\right\|-\left\|f^{\prime}\right\|\right) d t \\
& =\lim _{\alpha \rightarrow 0} \frac{1}{\alpha} \int_{0}^{1} \frac{\left\|f^{\prime}+\alpha h^{\prime}\right\|^{2}-\left\|f^{\prime}\right\|^{2}}{\left\|f^{\prime}+\alpha h^{\prime}\right\|+\left\|f^{\prime}\right\|} d t=\int_{0}^{1} \frac{\left\langle f^{\prime}, h^{\prime}\right\rangle}{\left\|f^{\prime}\right\|} d t
\end{aligned}
$$

Integrating by parts,

$$
\phi^{\prime}(f) h=\int_{0}^{1}\left\langle-\left(\frac{f^{\prime}}{\left\|f^{\prime}\right\|}\right)^{\prime}, h\right\rangle d t=\left\langle-\left(\frac{f^{\prime}}{\left\|f^{\prime}\right\|}\right)^{\prime}, h\right\rangle_{\left(L_{2}[0,1]\right)^{n}},
$$

so that, using (2),

$$
\nabla \phi(f)=-\left(\frac{f^{\prime}}{\left\|f^{\prime}\right\|}\right)^{\prime}=-\left\|f^{\prime}\right\| \kappa N
$$

As one would expect, a critical point of $\phi$ has zero curvature. The minimization problem does not have a unique solution, however, because the line segment does not have a unique parameterization. Consider a steepest descent method for minimizing $\phi$. The curves evolve in the negative gradient direction which, using the $L_{2}$ gradient, is toward the center of curvature at each point on the curve. This seems to make sense. However, the parameter-dependent scale factor $\left\|f^{\prime}\right\|$ is not appropriate and, more importantly, the $L_{2}$ gradient lacks smoothness. Each descent step reduces the number of continuous derivatives by two.

Equation (3) suggests the following inner product that depends on $f$ but not its parameterization:

$$
\langle g, h\rangle_{f} \equiv \int_{0}^{1} \frac{\left\langle g^{\prime}, h^{\prime}\right\rangle}{\left\|f^{\prime}\right\|} d t \quad \forall g, h \in \mathcal{C}_{o} .
$$

Positivity follows from the restriction to $\mathcal{C}_{o}$. To show that the inner product is intrinsic to the geometry of the curve, we can express it in terms of Stieltjes integrals:

$$
\langle g, h\rangle_{f}=\int_{0}^{1} \frac{\left\langle g^{\prime}(t), h^{\prime}(t)\right\rangle}{s^{\prime}(t)^{2}} s^{\prime}(t) d t=\int_{0}^{s(1)}\left\langle\frac{d g}{d s}, \frac{d h}{d s}\right\rangle d s .
$$

In order to demonstrate the relationship of our formulation with the standard Sobolev gradient setup, define a first-order differential operator $D_{f}$ by

$$
D_{f} h \equiv \frac{h^{\prime}}{\sqrt{s^{\prime}}} \quad \forall h \in \mathcal{C}_{o} .
$$

Then the adjoint operator $D_{f}^{*}$ is defined by

$$
\begin{aligned}
\left\langle h, D_{f}^{*} k\right\rangle_{\left(L_{2}[0,1]\right)^{n}} & =\left\langle D_{f} h, k\right\rangle_{\left(L_{2}[0,1]\right)^{n}}=\int_{0}^{1} \frac{\left\langle h^{\prime}, k\right\rangle}{\sqrt{s^{\prime}}} d t \\
& =\int_{0}^{1}\left\langle h,-\left(\frac{k}{\sqrt{s^{\prime}}}\right)^{\prime}\right\rangle d t=\left\langle h,-\left(\frac{k}{\sqrt{s^{\prime}}}\right)^{\prime}\right\rangle_{\left(L_{2}[0,1]\right)^{n}}
\end{aligned}
$$


for all $k, h \in \mathcal{C}_{o}$ so that

$$
D_{f}^{*} k=-\left(\frac{k}{\sqrt{s^{\prime}}}\right)^{\prime},
$$

and the negative $f$ Laplacian $L_{f} \equiv D_{f}^{*} D_{f}$ is given by

$$
L_{f}(h)=D_{f}^{*} D_{f} h=-\left(\frac{h^{\prime}}{s^{\prime}}\right)^{\prime} .
$$

The Fréchet derivative $\phi^{\prime}(f)$ is a bounded linear functional on $\mathcal{C}_{o}$ with the $f$ inner product as well as the $L_{2}$ inner product. By the Riesz Representation Theorem it is therefore uniquely represented by the $f$ gradient $g \equiv \nabla_{f} \phi(f) \in \mathcal{C}_{o}$. Thus, for all $h \in \mathcal{C}_{o}$,

$$
\begin{aligned}
\phi^{\prime}(f) h & =\langle\nabla \phi(f), h\rangle_{\left(L_{2}[0,1]\right)^{n}}=\langle g, h\rangle_{f} \\
& =\int_{0}^{1} \frac{\left\langle g^{\prime}, h^{\prime}\right\rangle}{s^{\prime}} d t=\left\langle D_{f} g, D_{f} h\right\rangle_{\left(L_{2}[0,1]\right)^{n}} \\
& =\left\langle D_{f}^{*} D_{f} g, h\right\rangle_{\left(L_{2}[0,1]\right)^{n}}
\end{aligned}
$$

so that

$$
\nabla \phi(f)=D_{f}^{*} D_{f} g=L_{f}(g) .
$$

Denote by $P_{o}$ the orthogonal projection onto the space of parametric curves $h$ that satisfy homogeneous end conditions $h(0)=h(1)=0$. Then the restriction of $P_{o} L_{f}$ to $\mathcal{C}_{o}$ is invertible, and its inverse serves as a smoothing operator for the gradient:

$$
\nabla_{f} \phi(f)=\left(P_{o} L_{f \mid \mathcal{C}_{o}}\right)^{-1} \nabla \phi(f)
$$

Since the Sobolev gradient $g$ is based on a metric that varies with $f$, a descent method using $g$ is, like a quasi-Newton method, a variable metric method.

For more general problems the trick to choosing the right inner product on the tangent space at $f$ is to retain independence from the parameterization by using derivatives with respect to arc length along $f$, and make the highest order of differentiation agree with that of the functional whose critical points are sought. When higher order derivatives are involved there remains some flexibility in choosing their relative weights. The weights used in the inner product need not agree with those appearing in the functional. The weights in the functional define the properties of the solution curve, while those in the inner product affect computational efficiency. Placing more weight on the low-order derivatives improves the condition number of the smoothing operator but may make it less effective, requiring more descent steps for convergence. Optimal weights are problem-dependent.

Returning to the curve-length problem, let $u=f-g$, corresponding to a single step of steepest descent with step-length 1 . The following trivial but surprising theorem shows that $u$ is the solution to the minimization problem.

Theorem 2.1 Let $u=f-g$ for $g=\nabla_{f} \phi(f)$. Then $\nabla \phi(u)=0$. 
proof. By $(4),(6)$, and $(7), L_{f}(f)$ and $L_{f}(g)$ are both equal to $\nabla \phi(f)$, so that

$$
-L_{f}(u)=\left(\frac{u^{\prime}}{s^{\prime}}\right)^{\prime}=\frac{s^{\prime} u^{\prime \prime}-s^{\prime \prime} u^{\prime}}{\left(s^{\prime}\right)^{2}}=0,
$$

implying that $u^{\prime \prime}$ and $u^{\prime}$ have the same direction, and $u$ therefore has zero curvature.

The following theorem characterizes the solution $u$ more precisely as a line segment parameterized by the arc length along the initial curve $f$.

Theorem 2.2 For $u=f-g, g=\nabla_{f} \phi(f) \in \mathcal{C}_{o}$,

$$
u(t)=f(0)+\frac{s(t)}{s(1)}[f(1)-f(0)] .
$$

proof. Let $\left(g^{\prime}-f^{\prime}\right) / s^{\prime}=c$, for constant $c$. Then $g^{\prime}=f^{\prime}+c s^{\prime}$, implying that

$$
g(t)=\int_{0}^{t} g^{\prime}=\int_{0}^{t}\left(f^{\prime}+c s^{\prime}\right)=f(t)-f(0)+c s(t),
$$

and $g(1)=f(1)-f(0)+c s(1)=0$, so that $c=[f(0)-f(1)] / s(1)$.

An alternative inner product is defined by $\langle g, h\rangle_{H(f)}=\langle H(f) g, h\rangle_{\left(L_{2}[0,1]\right)^{n}}$, when the Hessian $H(f)$ is positive definite on $\mathcal{C}_{o}$. In general the curve Laplacian $L_{f}$ is a preconditioner whose effectiveness depends on how close it is to $H(f)$. In order to obtain an expression for the Hessian, we compute the second derivative 
at $f$ in the directions $h, k \in \mathcal{C}_{o}$. Using (3),

$$
\begin{aligned}
& \phi^{\prime \prime}(f) h k=\int_{0}^{1}\langle k, H(f) h\rangle d t=\lim _{\alpha \rightarrow 0} \frac{1}{\alpha}\left[\phi^{\prime}(f+\alpha k) h-\phi^{\prime}(f) h\right] \\
& =\lim _{\alpha \rightarrow 0} \frac{1}{\alpha} \int_{0}^{1} \frac{\left\langle f^{\prime}+\alpha k^{\prime}, h^{\prime}\right\rangle}{\left\|f^{\prime}+\alpha k^{\prime}\right\|}-\frac{\left\langle f^{\prime}, h^{\prime}\right\rangle}{\left\|f^{\prime}\right\|} d t \\
& =\lim _{\alpha \rightarrow 0} \frac{1}{\alpha} \int_{0}^{1} \frac{\left\|f^{\prime}\right\|\left\langle f^{\prime}+\alpha k^{\prime}, h^{\prime}\right\rangle-\left\|f^{\prime}+\alpha k^{\prime}\right\|\left\langle f^{\prime}, h^{\prime}\right\rangle}{\left\|f^{\prime}+\alpha k^{\prime}\right\|\left\|f^{\prime}\right\|} d t \\
& =\int_{0}^{1} \frac{\left\langle k^{\prime}, h^{\prime}\right\rangle}{\left\|f^{\prime}\right\|}+\lim _{\alpha \rightarrow 0} \frac{1}{\alpha} \int_{0}^{1} \frac{\left\langle f^{\prime}, h^{\prime}\right\rangle\left(\left\|f^{\prime}\right\|-\left\|f^{\prime}+\alpha k^{\prime}\right\|\right)}{\left\|f^{\prime}+\alpha k^{\prime}\right\|\left\|f^{\prime}\right\|} d t \\
& =\int_{0}^{1}\left[\frac{\left\langle k^{\prime}, h^{\prime}\right\rangle}{\left\|f^{\prime}\right\|}-\frac{\left\langle f^{\prime}, h^{\prime}\right\rangle\left\langle f^{\prime}, k^{\prime}\right\rangle}{\left\|f^{\prime}\right\|^{3}}\right] d t \\
& =\int_{0}^{1}\left[\left\langle k^{\prime}, h^{\prime}\right\rangle-\left\langle\Delta \mathbf{f}, h^{\prime}\right\rangle\left\langle\Delta \mathbf{f}, k^{\prime}\right\rangle\right] / s^{\prime} d t \\
& =\int_{0}^{1}\left\langle k^{\prime}, h^{\prime}-\left\langle\Delta \mathbf{f}, h^{\prime}\right\rangle \Delta \mathbf{f}\right\rangle / s^{\prime} d t=\int_{0}^{1}\left\langle k^{\prime},\left(I-\Delta \mathbf{f} \Delta \mathbf{f}^{T}\right) h^{\prime}\right\rangle / s^{\prime} d t \\
& =\int_{0}^{1}\left\langle\frac{k^{\prime}}{\sqrt{s^{\prime}}},\left(I-\Delta \mathbf{f} \Delta \mathbf{f}^{T}\right) \frac{h^{\prime}}{\sqrt{s^{\prime}}}\right\rangle d t \\
& =\int_{0}^{1}\left\langle D_{f} k,\left(I-\Delta \mathbf{f} \Delta \mathbf{f}^{T}\right) D_{f} h\right\rangle d t \\
& =\int_{0}^{1}\left\langle k, D_{f}^{*}\left(I-\Delta \mathbf{f} \Delta \mathbf{f}^{T}\right) D_{f} h\right\rangle d t
\end{aligned}
$$

where $\Delta \mathbf{f}(t) \equiv f^{\prime}(t) /\left\|f^{\prime}(t)\right\|$ is the unit tangent vector at $f(t)$. The Hessian is thus

$$
H(f)=D_{f}^{*}\left(I-\Delta \mathbf{f} \Delta \mathbf{f}^{T}\right) D_{f} .
$$

This operator differs from $L_{f}$ only in the term $I-\Delta \mathbf{f} \Delta \mathbf{f}^{T}$ which projects onto the orthogonal complement of $\Delta \mathbf{f}$. If we replace $L_{f}$ by $H(f)$ in a steepest descent method, we have a damped Newton iteration. The following theorem shows that such a method is doomed to failure: the linear systems become increasingly ill-conditioned as the iteration proceeds.

Theorem 2.3 The Hessian of $\phi$ is singular at a critical point.

proof. A critical point $f$ is a parameterized line segment with constant unit tangent vector $\Delta \mathbf{f}$. Let $k(t)=\sin (2 \pi t) \Delta \mathbf{f}$. Then $k$ is a nonzero element of $\mathcal{C}_{o}$, but $D_{f} k$ has direction $\Delta \mathbf{f}$ and hence lies in the null space of $I-\Delta \mathbf{f} \Delta \mathbf{f}^{T}$, so that $k$ is in the null space of $H(f)$.

The singular Hessian is unusual. More typical behavior occurs in the problem of minimization of surface area. The Hessian of that functional, rather than being singular at a critical point, is positive definite only in the vicinity of a local minimum. In treating that problem we found it advantageous to switch 
from a Sobolev gradient descent method to a Newton iteration when the approximate solution was accurate enough for the Hessian to be positive definite. In some cases, however, we encountered numerical difficulties associated with nonuniqueness of the parameterization of critical points. That problem was solved by using an alternative to the surface area functional. The analogous alternative for curve length is the following parameter-dependent functional:

$$
\psi(f)=\frac{1}{2} \int_{0}^{1}\left\|f^{\prime}(t)\right\|^{2} d t
$$

The negative $L_{2}$ gradient, $-\nabla \psi(f)=f^{\prime \prime}$, is the acceleration vector, and with the endpoint constraints, $\psi$ is therefore uniquely minimized by the constantspeed line segment $f(t)=f(0)+t[f(1)-f(0)]$. Using $D h=h^{\prime}$, the negative Laplacian and the Hessian of $\psi$ are both defined by $D^{*} D h=-h^{\prime \prime}$ so that the Sobolev gradient descent method with step-length 1 is a Newton iteration, and converges in one iteration. Since $\psi$ is quadratic, this is no surprise. We use a discretization of $\psi$ as a regularization term for constructing minimum variation curves ([4]). It not only serves as a tension factor, preventing extraneous loops, but also helps to maintain uniformly distributed vertices.

\section{Discrete Minimum-length Curves}

We now consider the discretized minimum curve-length problem. In order to keep the notation simple, we retain some of the same symbols used in the function space setting. Although not significant for the simple problem treated here, a key idea is to represent a curve by an ordered sequence of discrete vertices. Then segment arc lengths are distances between vertices, and derivatives with respect to arc length are approximated by simple divided differences. The total curvature, for example, is a sum of squared second differences scaled by segment lengths. With a finite element formulation, on the other hand, the total curvature would be represented by a complicated formula involving a parameter $t$, and would require a high-order quadrature method to control the discretization error. To reiterate, there is no need to explicitly discretize the domain $[0,1]$. We implicitly assume a constant time interval $\Delta t$ associated with each segment so that constant speed corresponds to uniformly distributed vertices.

A discrete curve $\mathbf{f}$ is a sequence of $m+1$ vertices $\mathbf{f}_{i} \in \mathbf{R}^{n}, i=0, \ldots, m, m \geq 2$, where adjacent vertices are distinct, and $\mathbf{f}_{0}$ and $\mathbf{f}_{m}$ are distinct fixed endpoints. One might think of $\mathbf{f}$ as the polygonal curve (piecewise linear interpolant) associated with the vertices, but it should not be identified with a $C^{0}$ parametric spline curve. Only the endpoints are control points. In general, there are many more vertices than control points, and there are discretized derivative vectors along with the vertices. The sequence of vertices should be thought of as a discrete representation of a $C^{2}$ parametric curve.

Denote segment lengths by $\Delta s_{i}$, unit tangent vectors by $\Delta \mathbf{f}_{i}$, and vertex 
normal curvature vectors by $\Delta^{2} \mathbf{f}_{i}$ :

$$
\begin{aligned}
\Delta s_{i} & =\left\|\mathbf{f}_{i}-\mathbf{f}_{i-1}\right\| \quad(i=1, \ldots, m), \\
\Delta \mathbf{f}_{i} & =\frac{\mathbf{f}_{i}-\mathbf{f}_{i-1}}{\Delta s_{i}} \quad(i=1, \ldots, m), \\
\Delta^{2} \mathbf{f}_{i} & =\frac{\Delta \mathbf{f}_{i+1}-\Delta \mathbf{f}_{i}}{\Delta a_{i}} \quad(i=1, \ldots, m-1),
\end{aligned}
$$

where $\Delta a_{i}=\left(\Delta s_{i}+\Delta s_{i+1}\right) / 2$ is the portion of arc length associated with vertex $\mathbf{f}_{i}$. We again denote by $\mathcal{C}_{o}$ the set of perturbations for $\mathbf{f}$ which preserve the endpoint values - $(m+1)$-vectors of vertices $\mathbf{h} \in\left(\mathbf{R}^{n}\right)^{m+1}$ such that $\mathbf{h}_{0}=\mathbf{h}_{m}=\mathbf{0}$. The discretized curve length is then

$$
\phi(\mathbf{f})=\sum_{i=1}^{m} \Delta s_{i},
$$

and the ordinary gradient $\nabla \phi(\mathbf{f}) \in \mathcal{C}_{o}$ has component $n$-tuples

$$
\nabla \phi_{i}=\frac{\partial \phi}{\partial \mathbf{f}_{i}}=\Delta \mathbf{f}_{i}-\Delta \mathbf{f}_{i+1},
$$

for $i=1, \ldots, m-1$. Note that $\nabla \phi_{i}=-\Delta a_{i} \Delta^{2} \mathbf{f}_{i}$ so that the negative gradient at $\mathbf{f}_{i}$ has the direction of the normal curvature vector as in the continuous case (4). Again, critical points are characterized by zero curvature. Note also that the discretization of $\nabla \phi(f)$ as defined in (4) is $\nabla \phi_{i}=\left(\Delta \mathbf{f}_{i}-\Delta \mathbf{f}_{i+1}\right) / \Delta t$, and hence the ordinary gradient of the discretized functional is not the discretized $L_{2}$ gradient. However, it has the same zeros (even when $\Delta t_{i}$ is not constant).

Now define a discrete differential operator $D_{f}$ by

$$
\left(D_{f} \mathbf{h}\right)_{i} \equiv \frac{\mathbf{h}_{i}-\mathbf{h}_{i-1}}{\sqrt{\Delta s_{i}}} \quad(i=1, \ldots, m)
$$

for $\mathbf{h} \in \mathcal{C}_{o}$. For $m=4$, the matrix is

$$
D_{f}=\left(\begin{array}{ccccc}
-1 / \sqrt{\Delta s_{1}} & 1 / \sqrt{\Delta s_{1}} & 0 & 0 & 0 \\
0 & -1 / \sqrt{\Delta s_{2}} & 1 / \sqrt{\Delta s_{2}} & 0 & 0 \\
0 & 0 & -1 / \sqrt{\Delta s_{3}} & 1 / \sqrt{\Delta s_{3}} & 0 \\
0 & 0 & 0 & -1 / \sqrt{\Delta s_{4}} & 1 / \sqrt{\Delta s_{4}}
\end{array}\right),
$$

and the negative Laplacian is

$$
L_{f} \equiv D_{f}^{T} D_{f}=\left(\begin{array}{ccccc}
\frac{1}{\Delta s_{1}} & -\frac{1}{\Delta s_{1}} & 0 & 0 & 0 \\
-\frac{1}{\Delta s_{1}} & \frac{1}{\Delta s_{1}}+\frac{1}{\Delta s_{2}} & -\frac{1}{\Delta s_{2}} & 0 & 0 \\
0 & -\frac{1}{\Delta s_{2}} & \frac{1}{\Delta s_{2}}+\frac{1}{\Delta s_{3}} & -\frac{1}{\Delta s_{3}} & 0 \\
0 & 0 & -\frac{1}{\Delta s_{3}} & \frac{1}{\Delta s_{3}}+\frac{1}{\Delta s_{4}} & -\frac{1}{\frac{\Delta s_{4}}{\Delta s_{4}}} \\
0 & 0 & 0 & \frac{1}{\Delta s_{4}}
\end{array}\right),
$$

where $L_{f}$ is applied to an element $\mathbf{h} \in \mathcal{C}_{o}$ by applying it to each of the $n$ $(m+1)$-vectors associated with the components. If components of $\mathbf{h}$ are stored 
contiguously, the zeros and ones in $L_{f}$ may be interpreted as order- $n$ matrices. $L_{f}$ approximates a Neumann Laplacian, and has the constant vectors in its null space. We restrict $L_{f}$ to $\mathcal{C}_{o}$ and follow its application with projection onto $\mathcal{C}_{o}$ by simply omitting the first and last columns, and the first and last rows, respectively. Then we have a symmetric positive-definite tridiagonal matrix $P_{o} L_{f \mid \mathcal{C}_{o}}$ which we use in the discrete version of (8) to compute the Sobolev gradient. Note that we solve $n$ linear systems with the same order- $(m-1)$ matrix. The following theorem shows that, as in the continuous case, one step of steepest descent with the Sobolev gradient and step-length 1 produces the solution.

Theorem 3.1 For any discrete curve $\mathbf{f}, \mathbf{f}-\left(P_{o} L_{f \mid \mathcal{C}_{o}}\right)^{-1} \nabla \phi(\mathbf{f})$ is a parametric line segment with endpoints $\mathbf{f}_{0}$ and $\mathbf{f}_{m}$ and with the same vertex distribution as the initial curve $f$.

proof. Let $\mathbf{l}=\mathbf{f}-\left(P_{o} L_{f \mid \mathcal{C}_{o}}\right)^{-1} \nabla \phi(\mathbf{f})$. Then $\nabla \phi(\mathbf{f})=\left(P_{o} L_{f \mid \mathcal{C}_{o}}\right)(\mathbf{f}-\mathbf{l})$; i.e., for $i=1, \ldots, m-1$,

$$
\begin{aligned}
\Delta \mathbf{f}_{i}-\Delta \mathbf{f}_{i+1} & =-\frac{1}{\Delta s_{i}}(\mathbf{f}-\mathbf{l})_{i-1}+\left(\frac{1}{\Delta s_{i}}+\frac{1}{\Delta s_{i+1}}\right)(\mathbf{f}-\mathbf{l})_{i}-\frac{1}{\Delta s_{i+1}}(\mathbf{f}-\mathbf{l})_{i+1} \\
& =\frac{(\mathbf{f}-\mathbf{l})_{i}-(\mathbf{f}-\mathbf{l})_{i-1}}{\Delta s_{i}}-\frac{(\mathbf{f}-\mathbf{l})_{i+1}-(\mathbf{f}-\mathbf{l})_{i}}{\Delta s_{i+1}} \\
& =\Delta \mathbf{f}_{i}-\Delta \mathbf{f}_{i+1}-\frac{\mathbf{l}_{i}-\mathbf{l}_{i-1}}{\Delta s_{i}}+\frac{\mathbf{l}_{i+1}-\mathbf{l}_{i}}{\Delta s_{i+1}}
\end{aligned}
$$

and hence $\left(\mathbf{l}_{i+1}-\mathbf{l}_{i}\right) / \Delta s_{i+1}-\left(\mathbf{l}_{i}-\mathbf{l}_{i-1}\right) / \Delta s_{i}=\mathbf{0}$. Thus, all segments of $\mathbf{l}$ are collinear, and $\left\|\mathbf{l}_{i+1}-\mathbf{l}_{i}\right\| /\left\|\mathbf{l}_{i}-\mathbf{l}_{i-1}\right\|=\left\|\mathbf{f}_{i+1}-\mathbf{f}_{i}\right\| /\left\|\mathbf{f}_{i}-\mathbf{f}_{i-1}\right\|$ for $i=1, \ldots, m-$ $1 \square$.

The Hessian of $\phi$ at $\mathbf{f}$ is the block tridiagonal matrix with the following order- $n$ blocks in row $i$ :

$$
\begin{aligned}
H_{i, i-1} & =\frac{\partial^{2} \phi}{\partial \mathbf{f}_{i} \partial \mathbf{f}_{i-1}}=-\frac{I-\Delta \mathbf{f}_{i} \mathbf{f}_{i}^{T}}{\Delta s_{i}} \\
H_{i, i} & =\frac{\partial^{2} \phi}{\partial \mathbf{f}_{i}^{2}}=\frac{I-\Delta \mathbf{f}_{i} \mathbf{f}_{i}^{T}}{\Delta s_{i}}+\frac{I-\Delta \mathbf{f}_{i+1} \mathbf{f}_{i+1}^{T}}{\Delta s_{i+1}} \\
H_{i, i+1} & =\frac{\partial^{2} \phi}{\partial \mathbf{f}_{i} \partial \mathbf{f}_{i+1}}=-\frac{I-\Delta \mathbf{f}_{i+1} \mathbf{f}_{i+1}^{T}}{\Delta s_{i+1}}
\end{aligned}
$$

for $i=1, \ldots, m-1$. It is easily verified that $H(\mathbf{f})=D_{f}^{T} P D_{f}$, where $P$ is a block diagonal matrix with blocks $P_{i}=I-\Delta \mathbf{f}_{i} \Delta \mathbf{f}_{i}^{T}$, and $H(\mathbf{f})$ is singular when $\mathbf{f}$ is a discretized line segment with constant unit tangent vector $\Delta \mathbf{f}$. Any sequence of vertices beginning and ending at the origin and lying on the line defined by $\Delta \mathbf{f}$ is an element of $\mathcal{C}_{o}$ and lies in the null space of $H(\mathbf{f})$ in this case.

The discrete version of the quadratic functional $\psi$ is $\psi(\mathbf{f})=(1 / 2) \sum_{i=1}^{m} \Delta s_{i}^{2} /$ $\Delta t$, where $\Delta t=1 / m$ is the time interval associated with each curve segment, 
assumed constant as mentioned in the opening paragraph of this section. Since this constant has no affect on critical points, we use the definition

$$
\psi(\mathbf{f})=\frac{1}{2} \sum_{i=1}^{m} \Delta s_{i}^{2} .
$$

The gradient $\Delta \psi(\mathbf{f})$ has components

$$
\Delta \psi_{i}=\frac{\partial \psi}{\partial \mathbf{f}_{i}}=-\mathbf{f}_{i-1}+2 \mathbf{f}_{i}-\mathbf{f}_{i+1},
$$

and the Hessian of $\psi$ is the block tridiagonal matrix $(m-1$ order- $n$ blocks) with constant diagonal blocks $2 I$ and off-diagonal blocks $-I$. This matrix is also proportional to the negative Dirichlet Laplacian $L=D^{T} D$, where $D$ is the discrete first derivative operator defined by $(D \mathbf{h})_{i}=\left(\mathbf{h}_{i}-\mathbf{h}_{i-1}\right) / \Delta t$. One Newton iteration produces a zero of $\nabla \psi$ characterized by $\mathbf{f}_{i+1}-\mathbf{f}_{i}=\mathbf{f}_{i}-\mathbf{f}_{i-1}$ for $i=1, \cdots, m$.

\section{Test Results}

We tested three methods: steepest descent with the ordinary gradient, steepest descent with the discretized Sobolev gradient, and Newton's method, on the two problems: minimization of $\phi(\mathbf{f})$ and minimization of $\psi(\mathbf{f})$ defined by (10) and (11), respectively. In most cases we took the initial curve $\mathbf{f}$ to be a uniformly distributed sequence of $m=200$ vertices on the parametric cubic spline interpolant of $(0,0),(1,1),(0,1)$, and $(1,0)$ in that order - a self-intersecting curve with endpoints $(0,0)$ and $(1,0)$.

For the most part, the test runs merely serve as dramatic illustrations of the theorems. The Sobolev gradient method produces the discretized line segment (with accuracy close to machine precision) in a single iteration, as does Newton's method for minimizing $\psi$. Newton's method applied to $\phi$ failed to converge even when started with an initial estimate very close to the line segment. The most interesting result was the miserable performance of the standard method of steepest descent. For minimizing $\phi$ the method failed (was unable to further reduce the functional) far from the solution, both with a line search and with a small constant step-size (0.01). When applied to the quadratic functional $\psi$, the method converged to a line segment of length 1.001 but only with constant step-size at most 0.5 , and that required 39000 iterations in the best case.

The problem treated here is very special in that, by Theorem 3.1, the solution of a linear system (inverting the f-Laplacian) essentially produces the solution of a system of nonlinear equations $\nabla \phi(\mathbf{f})=\mathbf{0}$. On the other hand, the relative effectiveness of the Sobolev gradient method compared to a gradient descent method with the ordinary gradient is not atypical. Our experience with more challenging geometric problems is that the standard gradient descent methods fail completely due to the lack of smoothness in the discretized $L_{2}$ gradient while the Sobolev gradient method requires very few iterations for convergence - at most a few hundred, and often fewer than 10 . 


\section{References}

[1] A. Linnér and R. J. Renka, Discrete periodic geodesics in a surface, Experimental Math. 14, No. 2 (2005), 145-152.

[2] R. J. Renka and J. W. Neuberger, Minimal surfaces and Sobolev gradients, SIAM. J. Sci. Comput. 16 (1995), 1412-1427.

[3] R. J. Renka, Algorithm 828. DNSPLIN1: Discrete nonlinear spline interpolation, ACM Trans. Math. Softw. 29, No. 4 (2003), 1-11.

[4] R. J. Renka, Constructing fair curves and surfaces with a Sobolev gradient method, CAGD 21 (2004), 137-149.

[5] R. J. Renka, A Sobolev gradient method for construction of elastic curves in regular surfaces, J. Nonlinear Analysis: Theory, Methods and Applications 63 (2005), e1789-e1796.

[6] R. J. Renka, Shape-preserving interpolation by fair discrete $G^{3}$ space curves, CAGD 22, No. 8 (2005), 793-809. 